\title{
Synthetic Aperture Ultrasound Image Reconstruction
}

\author{
Mawia Ahmed Hassan \\ Biomedical Engineering Department, Sudan University of Science \& Technology \\ Khartoum, Sudan (www.sustech.edu)
}

\begin{abstract}
In this paper real data was used to test the utility of synthetic aperture (SA) ultrasound imaging to overcome the limitation of conventional ultrasound image which include decreasing the frame rate and single transmit focusing. The images are reconstructed by using different types of transmission and used to test the effect of signal to noise ratio (SNR). The results show that increasing the number of aperture elements improves the SNR. This means that overcoming the problem of low SNR can be achieved by replacing single element transmission by simultaneous excitation of multi-element subapertures. 24-tap FIR Hilbert transformed was designed with acceptable normalized RMSE with the analytical form of the signal. The images were reconstructed with and without the FIR Hilbert transform filter. Synthetic aperture imaging is shown to have potential for alleviating the problem of frame rate limitation and single transmit focusing. The results were compared to image reconstructed using linear array image reconstruction. On the other hand, it is also shown to have several problems associated with its practical implementation in terms of penetration depth, flow estimation, and implementation.
\end{abstract}

\section{General Terms}

Medical Imaging, Image Reconstruction, Digital Signal Processing.

\section{Keywords}

Synthetic aperture; liner array image reconstruction; Ultrasound imaging; Hilbert transform; digital filter

\section{INTRODUCTION}

Synthetic aperture techniques were originally conceived for radar systems in the 1950 s and were initially implemented using digital computers in the late 1970s and more advanced techniques were introduced in the late 1980s [1]. There are many similarities between Radar and ultrasound systems, but there are also very significant differences. A SA Radar system usually employs one transmitter and receiver, and the aperture in synthesized by moving the antenna over the region of interest in an airplane or satellite. In medical ultrasound, the array has a fixed number of elements and is usually stationary. The synthesizing is performed by acquiring data from parts of the array to reduce the amount of electronic channels. For Radar, the object is most often in the far-field of the array, whereas the object always is in the near-field of a medical ultrasound system, which complicates the reconstruction. Since the medical array is stationary, it is possible to repeat measurements rapidly, which is not the case for a SA Radar systems [12]. The position between the different elementsis also fixed in ultrasound, whereas the deviations from a straight flight path for airplane often have to be compensated for in Radar systems. A vital difference is also that the dynamic range in a Radar image is significantly less than the 40-80 dB dynamic range in ultrasound images.

Until the beginning of 1990, the idea of applying the SA imaging approach for medical ultrasound imaging had only been considered occasionally [2],[3],[4].

In this paper, instead of a focused beam, a single element or a group of elements are used to transmit spherical waves which insonate the entire field of view. In this way it is possible to obtain an image with just one pulse, with consequent increase in frame rate, albeit at lower spatial resolution than for conventional imaging. A series of low-resolution images is acquired, each for different positions of the transmit element(s) (see Figure 1). The images are summed to produce a final high resolution image [4],[5]. In recursive imaging, for an array of $\mathrm{N}$ elements the acquisition sequence is repeated. The first high-resolution frame is made from emissions 1 to $\mathrm{N}$, the second from emissions 2 to $\mathrm{N}+1$, the third from emissions 3 to $\mathrm{N}+2$ and so on. In this way a new highresolution image is produced after every emission, but there is temporal overlap between the images [5]. To overcome the problem with low SNR and impedance matching between the transducer and receiver circuit, the single element transmission was replaced by simultaneous excitation of a multi-element subaperture. The synthetic aperture technique was compared to image reconstructed using linear array image reconstruction.

\section{METHODOLOGY}

\subsection{The Beamforming}

Focusing was performed by finding the geometric distance from the transmitting element to the imaging point and back to the receiving element. Dividing this distance by the speed of sound $c$ gives the time instance $t_{p}(i, j)$ to take out the proper signal value for summation. For an image point $\overrightarrow{r_{b}}$ the time is, thus [6]:

$t_{p}(i, j)=\frac{\left|\overrightarrow{r_{b}}-\overrightarrow{r_{e}}(\mathrm{i})\right|+\left|\overrightarrow{r_{b}}-\overrightarrow{r_{r}}(\mathrm{j})\right|}{c}$. 


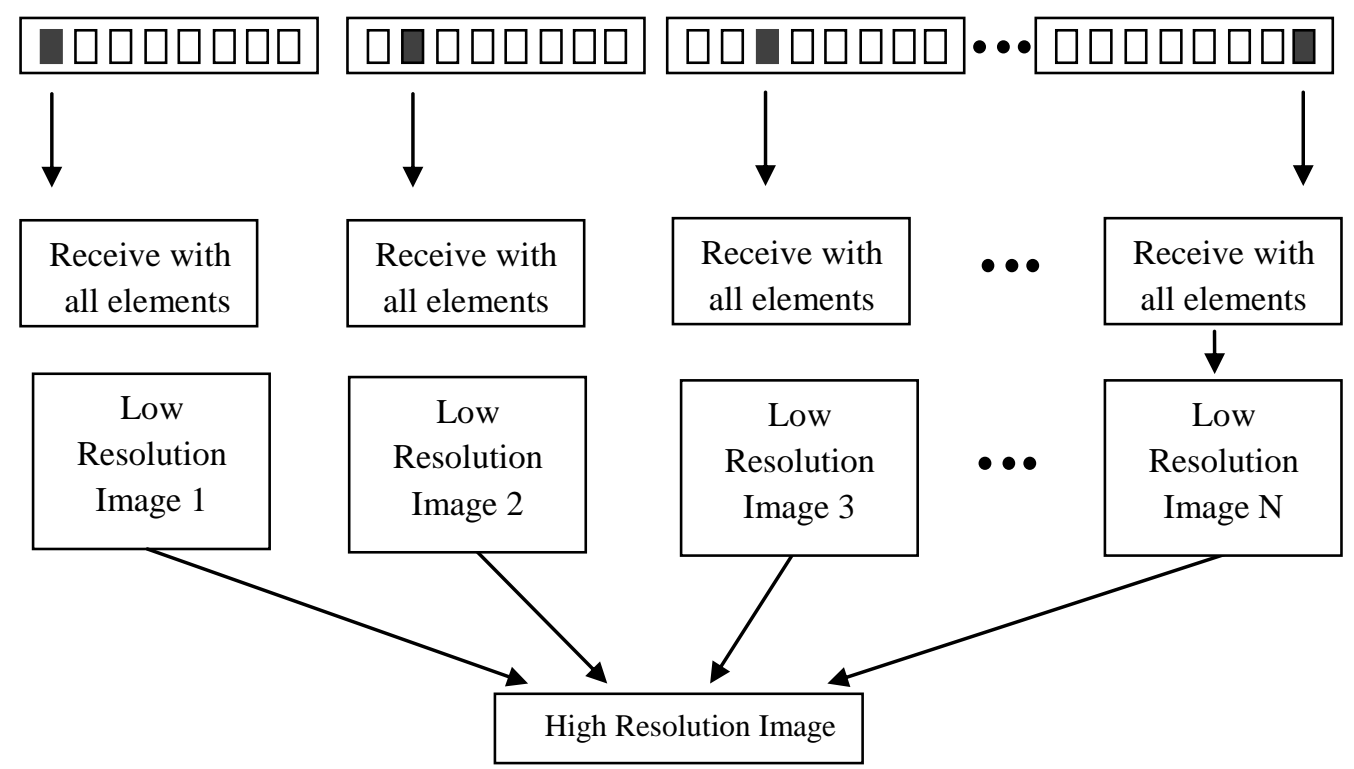

Fig 1: Synthetic aperture imaging

Here, $\overrightarrow{r_{e}}(i)$ denotes the position of the transmitting element $i$ and $\overrightarrow{r_{r}}(j)$ the receiving element $j$ 's position. This was done for every point in the resulting image to yield a low resolution image. Combining the low resolution images then results in a high resolution image, since fully dynamic focusing has been performed for all points in the image. The final focused signal $\mathrm{y}_{\mathrm{f}}\left(\overrightarrow{\mathrm{r}_{\mathrm{b}}}\right)$ is then [6]:

$$
y_{f}\left(\overrightarrow{r_{b}}\right)=\sum_{j=1}^{N} \sum_{i=1}^{M} a\left(t_{p}(i, j), i, j\right) y_{r}\left(t_{p}(i, j), i, j\right),
$$

wherey $_{r}(t, i, j)$ is the received signal for emission $i$ on element $j, a\left(t_{p}(i, j), i, j\right)$ is the weighting function (apodization) applied onto this signal, $\mathrm{N}$ is the number of transducer elements, and $\mathrm{M}$ is the number of emissions. The transmit focusing is, thus, synthesized by combining the low resolution images, and the focusing calculation makes the transmit focus dynamic for all points in the image. The focus is, therefore, both dynamic in transmit and receive and the highest possible resolution for delay-sum beamforcing is obtained everywhere in the image.

\subsection{Hilbert Transform Filter Design}

The analytic envelope of the signal was calculated as the square root of the sum of the squares of the real and quadrature components [7]. The most accurate way of obtaining the quadrature components was to pass the echo signal through a Hilbert transform [8], because it provides 90degree phase shift at all frequencies [9]. The impulse response of the Hilbert filter with length $\mathrm{N}$ (odd number) is defined as in [10]:

$$
h[n]= \begin{cases}\frac{2}{\pi} \frac{\sin ^{2}(\pi(n-\alpha) / 2)}{n-\alpha}, & n \neq \alpha, \\ 0, & n=\alpha\end{cases}
$$

$\alpha=(\mathrm{N}-1) / 2$. We chose the filter length equals $16,20,24,28$ and 32-tap with a Hamming window used to reduce the sidelobe effects. According to the normalized root mean square error (RMSE) between the designed FIR Hilbert filter and ideal Hilbert transform filter. The values of the RMSE for the five FIR filters were equaled $0.0109,0.0096,0.0092$, 0.0091 , and 0.0090 . We selected the 24-tap FIR filter because it provided a medium RMSE.

\subsection{Image Reconstruction}

The image was reconstructed using synthetic transmit aperture (STA). The effect of SNR was tested by using different types of transmission ( transmitted by one element received by all 128 elements, transmitted by 2 elements received by all 128 elements, transmitted by 4 elements received by all 128 elements, transmitted by 8 elements received by all 128 elements, transmitted by 16 elements received by all 128 elements, transmitted by 32 elements received by all 128 elements, transmitted by 64 elements received by all 128 elements, and transmitted by 128 elements received by all 128 elements) (Figure 2). To overcome the problem with low SNR the single element transmission was replaced by simultaneous excitation of a multi-element subaperture.

\section{RESULTS AND DISCUSSIONS}

\subsection{The Ultrasound data}

Real data obtained from the Biomedical Ultrasound Laboratory, University of Michigan was used [11]; the data set that was used to generate the results here is under "Acusonl7". The parameters for this data set are as follows: the number of channels was 128 channels, and the ADC sampling rate was 13.8889 MSPS. Linear shape transducer was used to acquire the data with center frequency of 3.5 $\mathrm{MHz}$, and element spacing of $0.22 \mathrm{~mm}$. Each ultrasonic Ascan was saved in a record consisted of $2048 \mathrm{RF}$ samples per line each represented in 2 bytes, and the signal averages was 8. The speed of the ultrasound in the phantom was 1480 $\mathrm{m} / \mathrm{sec}$. The data were acquired for phantom within 6 pins at different positions.

\subsection{Hilbert transform filter design}

As it mentioned in the methodologies, five lengths 16-, 20-, 24-, 28-, and 32-tap FIR Hilbert filter were used. Figure 3 showed the frequency response for each one. Figure 4(a) and (b) described the ideal analytical signal and ideal Hilbert frequency spectrum for 70 samples of ultrasound data. As it 


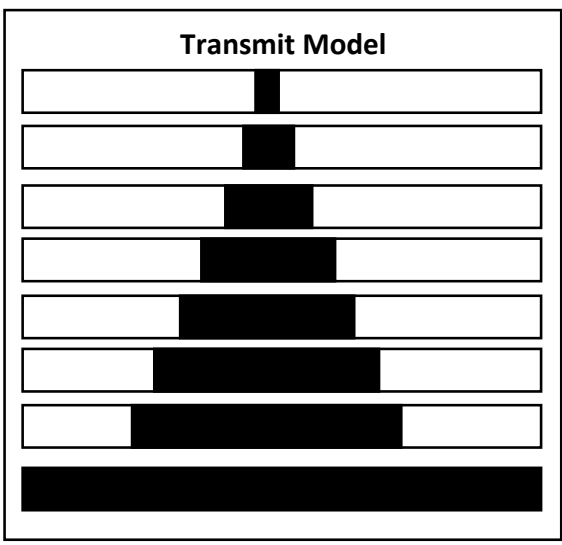

\begin{tabular}{|c|}
\hline Transmit Firing \\
1 Ele.(64) \\
2 Ele.(64,65) \\
4 Ele.(63:66) \\
8 Ele.(61:68) \\
16 Ele.(57:72) \\
32 Ele.(49:80) \\
64 Ele.(33:96) \\
128 Ele.(1:128) \\
\end{tabular}

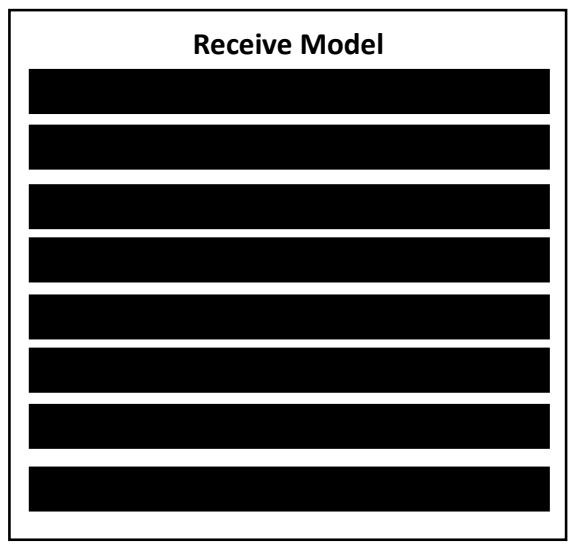

Fig 2: Synthetic aperture transmits and receive model

mentioned in the methodologies the normalized RMSE between the designed filter and ideal Hilbert transform filter of 24order FIR Hilbert provided a medium RMSE, so this filter was used for the simulation and implementation of the ultrasound data. Apply this designed Hilbert filter to the received echo line after delay and sum shown in Figure 5(a) compared to the ideal Hilbert filter in Figure 5 (b).

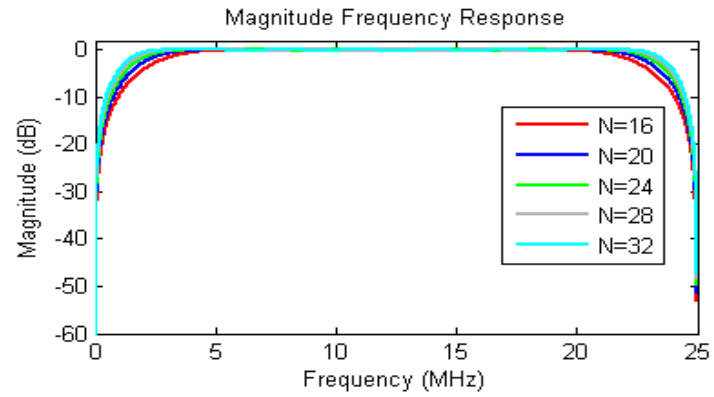

Fig 3: Magnitude frequency responses for FIR Hilbert filter with filter length equals 16, 20, 24, 28, and 32 .
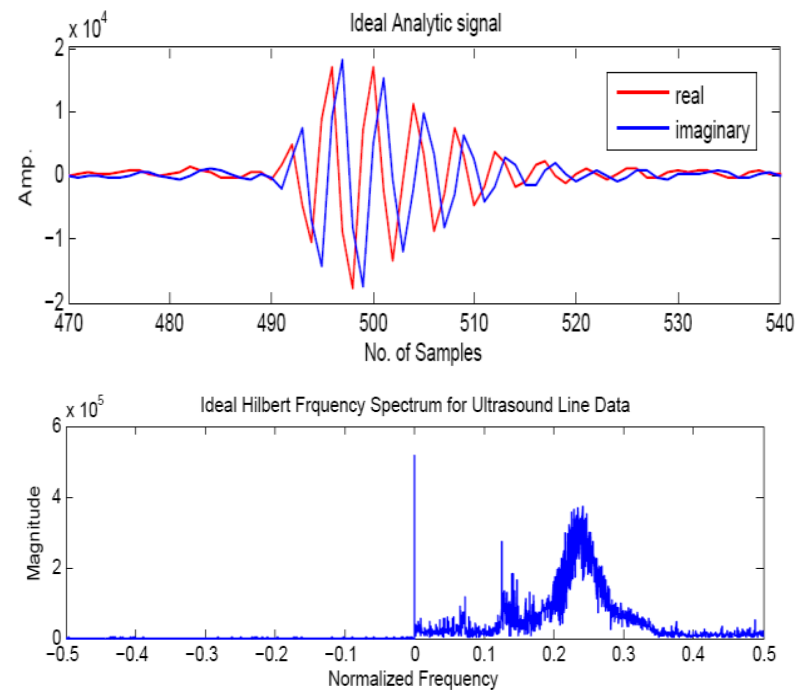

(b)

Fig 4: Ideal Hilbert transform filter. (a) Ideal analytical signal, (b) Ideal Hilbert frequency spectrum

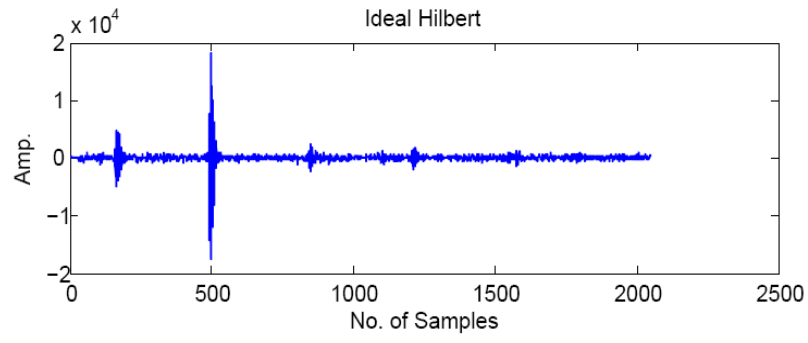

(a)

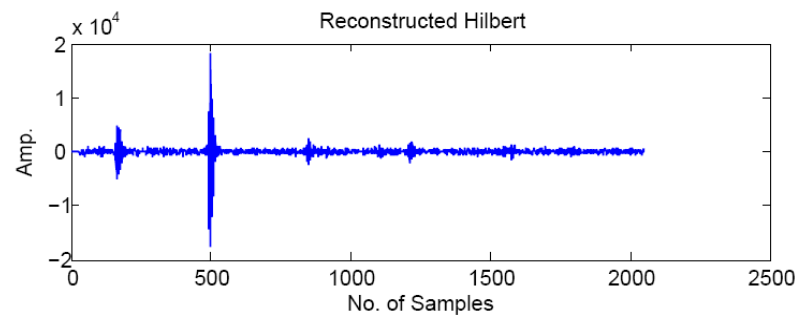

(b)

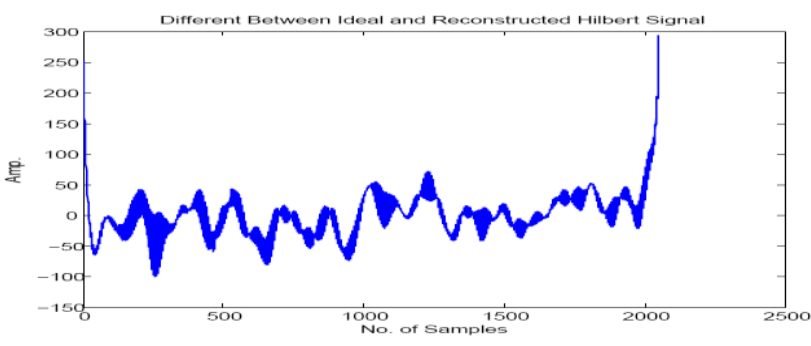

(c)

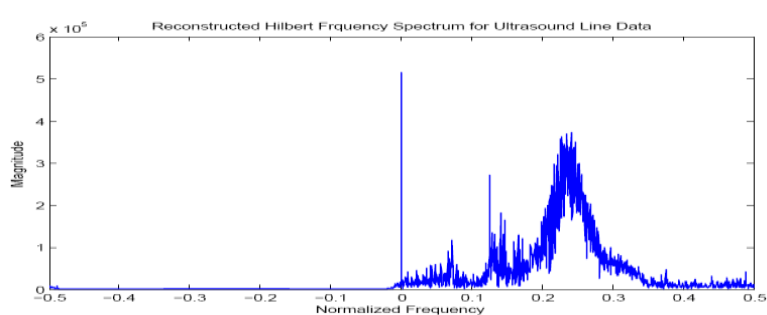

(d)

Fig 5: Hilbert filter apply to ultrasound line data. (a) Reconstructed FIR Hilbert, (b) Ideal Hilbert, (c) RMSE between reconstructed FIR and ideal Hilbert, (d) frequency spectrum of reconstructed FIR Hilbert. 
The RMSE between two signals is being shown in Figure 5 (c) and the value equal to $(0.3769)$ which mean that the two signals were near the same. Figure 5(d) described the frequency spectrum for ultrasound data. As can be shown the negative frequency was eliminated compared to the ideal Hilbert filter frequency spectrum (Figure 5(b)). When increase the order of the filter would be better (the longer the filter is, the narrower the bandwidth will be).

\subsection{STA image reconstruction}

Figure 6 shows the images reconstructed without Hilbert filter and Figure 7 shows the images reconstructed with FIR Hilbert filter using transmit model in Figure 2.

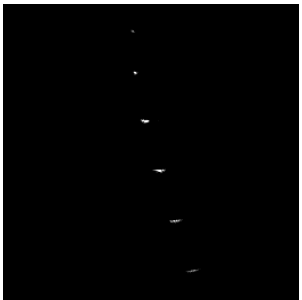

(a)

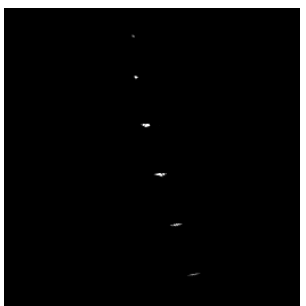

(c)

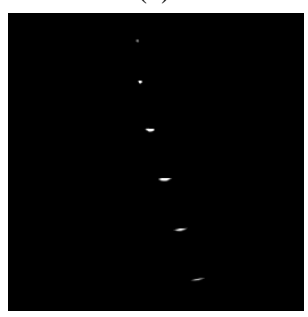

(e)

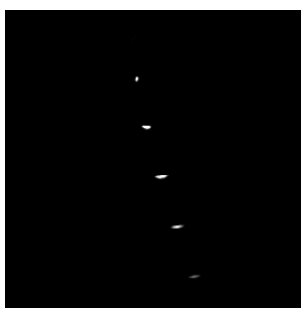

(g)

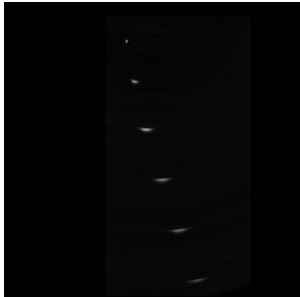

(b)

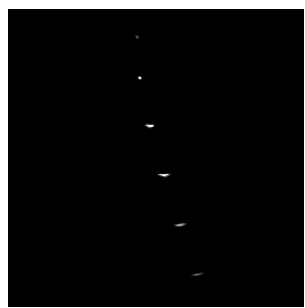

(d)

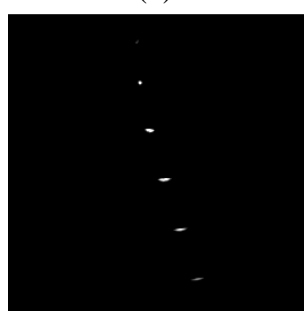

(f)

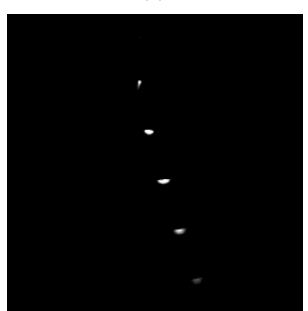

(h)
Fig 6: Image reconstructed without FIR Hilbert filter. (a) Transmitted by element no.1 and received by all 128 elements, (b) Transmitted by element no.64 and 65 and received by all 128 elements, (c) Transmitted by element no.63 to 66 and received by all 128 elements, (d)

Transmitted by element no.61 to 68 and received by all 128 elements, (e) Transmitted by element no.57 to 72 and received by all 128 elements, (f) Transmitted by element no.48 to 80 and received by all 128 elements, $(\mathrm{g})$ Transmitted by element no.33 to 96 and received by all 128 elements, (h) Transmitted by element no.1 to 128 and received by all 128 elements.

As can be shown the images reconstructed using Hilbert filter looking better than image without Hilbert filter. Also when we increased the number of aperture elements that improved the lateral resolution and the FOV.

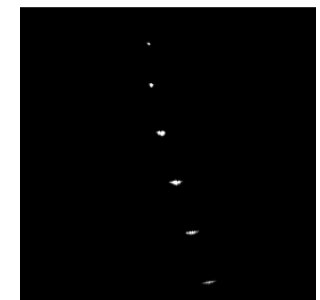

(a)

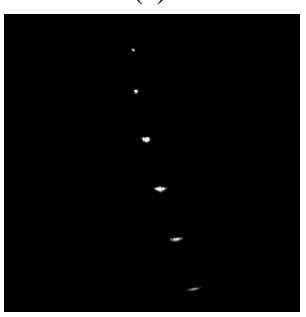

(c)

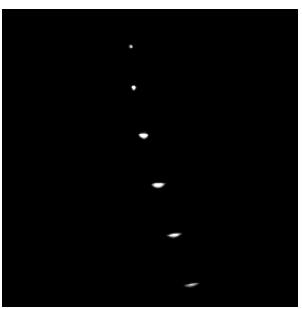

(e)

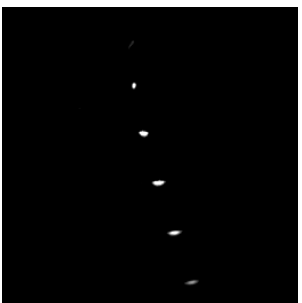

(g)

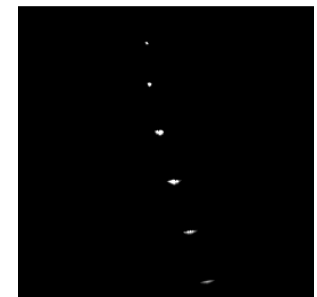

(b)

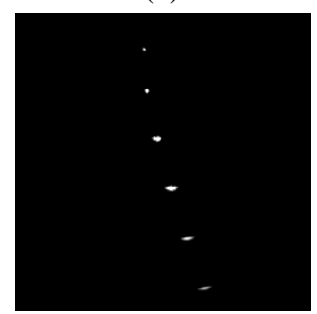

(d)

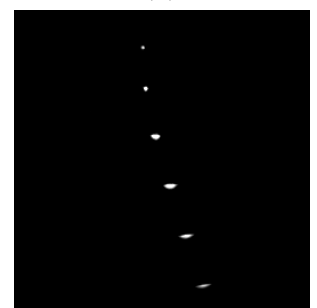

(f)

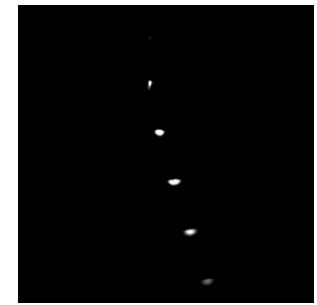

(h)
Fig 7: Image reconstructed with FIR Hilbert filter. (a) Transmitted by element no.1 and received by all 128 elements, (b) Transmitted by element no.64 and 65 and received by all 128 elements, (c) Transmitted by element no.63 to 66 and received by all 128 elements, (d)

Transmitted by element no.61 to 68 and received by all 128 elements, (e) Transmitted by element no.57 to 72 and received by all 128 elements, (f) Transmitted by element no.48 to 80 and received by all 128 elements, (g)

Transmitted by element no.33 to 96 and received by all 128 elements, (h) Transmitted by element no.1 to 128 and received by all 128 elements.

Figure 8 ( $a$ and $b$ ) shows the effect of SNR for SAT images when transmitted with 1-element, 2-elements, 4-elements, 8elements, 16-elements, 32- elements, 64-elements, 128elements and received with all 128 elements with and without using FIR Hilbert filter. The results show that when increasing the number of aperture elements it improved the SNR and envelope image gave better results than without it. Also the result is compared with image reconstructed using phase array image reconstruction [13] (raster point technique) as in Figure 9. The FOV in Figure 6 and 7 were better than Figure 9 also the lateral resolution. 


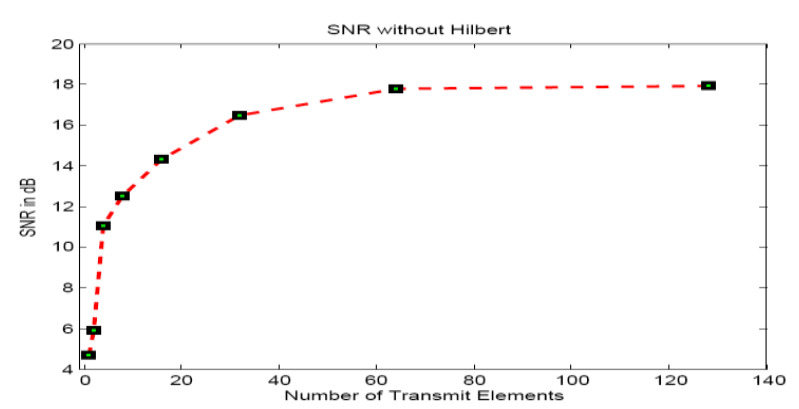

(a)

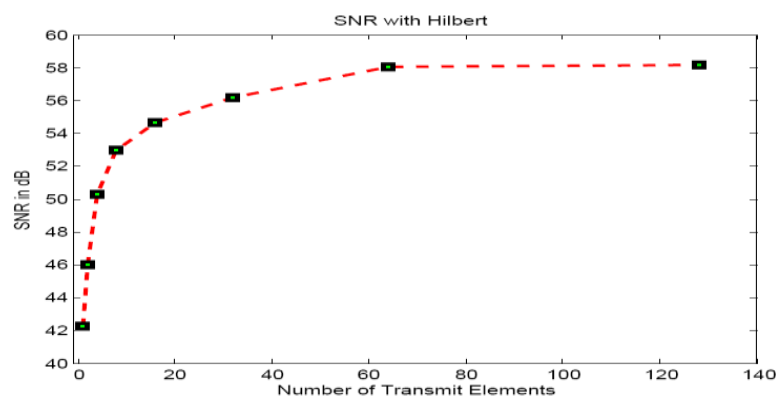

(b)

Fig. 8: SNR for STA images with 1,2,4,8,16,32,64, and 128 transmitted elements. (a) Without FIR Hilbert (b) With FIR Hilbert filter.

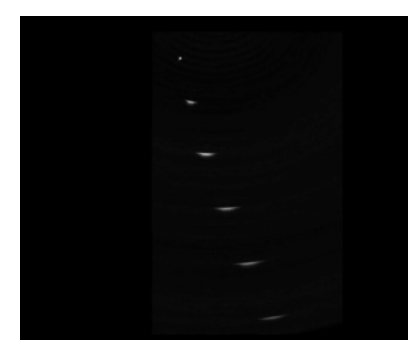

Fig 9: Image reconstructed using phase array image reconstruction (raster point technique).

Figure 10 show conventional linear array image reconstructed using aperture (Ap) equal to 1, 2,4,8,16,32, and 64. When the Ap size increased the lateral resolution was improved. However, the FOV was reduced compared to SA in Figure 6 and Figure 7.

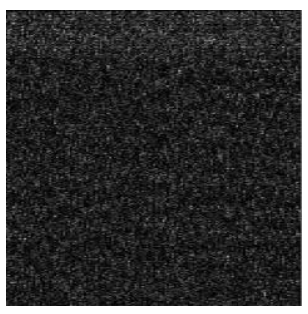

(a)

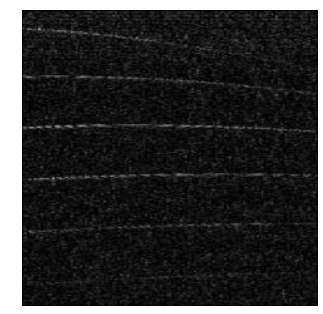

(b)

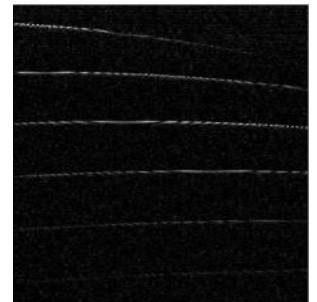

(c)

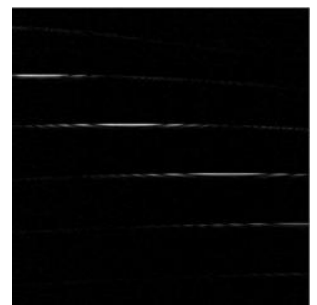

(e)

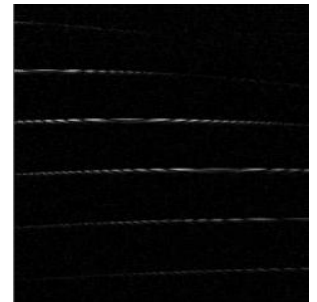

(d)

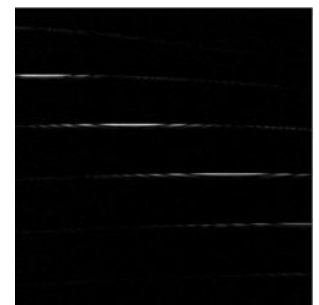

(f)

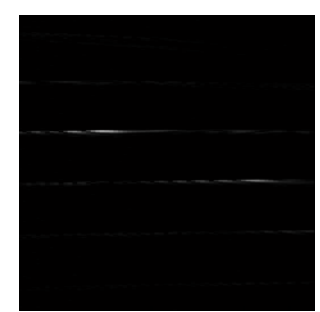

(g)

Fig 10: Linear array image reconstructed. (a) Using Ap=1, (b) Using Ap=2, (c) Using Ap=4, (d) Using Ap=8, (e) Using $A p=16$, (f) Using $A p=32$, (g) Using $A p=64$.

\section{CONCLUSIONS}

In this paper, the effect of SA ultrasound imaging was tested to overcome the limitation of conventional ultrasound. The images were reconstructed by using different types of transmission. The results showed that when increasing the number of aperture elements it improved the SNR. The images were reconstructed with and without the FIR Hilbert transform filter. The results were compared with image reconstruction using phase array image reconstruction (raster point technique) and conventional linear array image reconstructed using $\mathrm{Ap}=1,2,4,8,16,32$, and 64 . The SA was shown better results in FOV SNR and lateral resolution. Also SA imaging solved the problem of frame rate and single transmit focusing, but it also has several problems associated with it in terms of penetration depth, flow estimation, and implementation.

\section{REFERENCES}

[1] M. Soumekh 1999. Synthetic Aperture Radar Signal Processing with MATLAB Algorithms, John Wiley \& Sons, Inc., New York.

[2] J.J. Flaherty, K.R. Erikson, V.M. Lund 1967. Synthetic aperture ultrasound imaging systems. United States Patent, US 3,548-642.

[3] C.B. Burckhardt, P-A. Grandchamp, H. Hoffmann 1974. An experimental $2 \mathrm{MHz}$ synthetic aperture sonar system intended for medical use. IEEE Trans. Son. Ultrason. 21 (1), 1-6.

[4] K. Nagai 1985.A new synthetic-aperture focusing method for ultrasonic Bscan imaging by the fourier transform. IEEE Trans. Son. Ultrason. SU-32 (4), 531536. 
[5] P.Hoskins, K.Martin, A.Thrush 2010. Diagnostic Ultrasound Physics and Equipment. Cambridge University Press. Cambridge.

[6] J.A. Jensen, S.I. Nikolov, K.L. Gammelmark, and M.H.Pedersen 2006. Synthetic aperture ultrasound imaging. Ultrasonics. 44,e5-e15.

[7] J. O. Smith 2002. Mathematics of the Discrete Fourier Transform (DFT). Center for Computer Research in Music and Acoustics (CCRMA).Department of Music, Stanford University, Stanford, California.

[8] A. V. Oppenheim and R. W. Schafer 1989. DiscreteTime Signal Processing. NJ: Prentice-Hall, Englewood Cliffs.

[9] B.G. Tomov and J.A. Jensen 2005. Compact FPGABased Beamformer Using Oversampled 1-bit A/D Converters. IEEE Transactions on Ultrasonics, Ferroelectrics and Frequency Control, vol. 52, no. 5, 870-880.
[10] S. Sukittanon, S.G. Dame 2005. FIR Filtering in PSoC ${ }^{\text {TM }}$ with Application to Fast Hilbert Transform. Cypress Semiconductor Corp., Cypress Perform.

[11] M. O'Donnell and S.W. Flax 1988. Phase-aberration correction using signals from point reflectors and diffuse scatterers: measurements. IEEE Trans. Ultrason., Ferroelect., and Freq. Contr., vol. 35, no. 6,768-774.

[12] J. opretzka, M. Vogt and H.Ermert 2011. A highfrequency ultrasound imaging system combining limitedangle spatial compounding and model-based synthetic aperture focusing. IEEE Trans. Ultrason., Ferroelect., and Freq. Contr., vol. 58, no. 7, 1355-1365.

[13] Mawia A. H. and Yasser M.. K. 2013. Digital Signal Processing Methodologies for Conventional Digital Medical Ultrasound Imaging System. American Journal of Biomedical Engineering., vol. 3, no. 1, 14-30. 